Arbeitsweisungen und Arbeitsauflagen im Jugendstrafrecht · Kremerskothen 


\section{Schriften zum Jugendrecht und zur Jugendkriminologie}

herausgegeben von

Jörg M. Fegert, Wolfgang Heinz, Horst Viehmann, Reinhard Wiesner und Michael Walter

\section{BAND 3}




\title{
Arbeitsweisungen und Arbeitsauflagen im Jugendstrafrecht
}

\author{
Differenzierungsansäłze \\ in der Theorie und der Praxis \\ im Rhein-Neckar-Kreis
}

Heike Kremerskothen

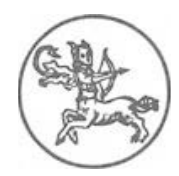

Centaurus Verlag \& Media UG 2001 
Die Autorin, geb. 1972, studierte Rechtswissenschaften an der Universitüt Heidelberg und promovierte 2001 an der Universitôt Konstanz. Sie ist als Richterin in der Hessischen Arbeitsgerichłsbarkeit tötig.

Die Deutsche Bibliothek - CIP-Einheitsaufnahme

Kremerskothen, Heike:

Arbeitsweisungen und Arbeitsauflagen im Jugendstrafrecht:

Differenzierungsansäłze in der Theorie und Praxis im Rhein-Neckar-Kreis /

Heike Kremerskothen. - Herbolzheim : Centaurus-Verl.GmbH \& Co. KG, 2001

(Schriften zum Jugendrecht und zur Jugendkriminologie ; Bd. 3)

Zugl.: Konstanz, Univ., Diss., 2001

ISBN 978-3-8255-0344-4

DOI 10.1007/978-3-86226-290-8

\section{ISSN 1434-3614}

Alle Rechte, insbesondere das Recht der Vervielföltigung und Verbreitung sowie der Übersetzung, vorbehatten. Kein Teil des Werkes darf in irgendeiner Form (durch Fotokopie. Mikrofilm oder ein anderes Verfahren) ohne schriftiche Genehmigung des Verlages reproduziert oder unter Verwendung elektronischer Systeme verarbeitet, vervielfältigt oder verbreitet werden.

(C) CENTAURUS Verlags-GmbH \& Co. KG, Herbolzheim 2001

Satz: Vorlage der Autorin

Umschlaggestalłung: DTP-Studio, Antje Walter, Lenzkirch 


\section{Vorwort}

Das hier vorgelegte Werk ist ein Versuch, die theoretischen Voraussetzungen der Abgrenzung von Arbeitsauflagen und Arbeitsweisungen sowie deren Umsetzung in die Praxis im Rhein-Neckar-Kreis darzustellen. Die Arbeit wurde im Wintersemester 2000/2001 bei der Juristischen Fakultät der Universität Konstanz eingereicht.

Ich möchte es nicht versäumen, all jenen zu danken, die mich in meiner Arbeit unterstützt haben. Meinem Doktorvater Herrn Prof. Dr. Wolfgang Heinz danke ich besonders herzlich für dessen wohlwollende Begleitung meiner Arbeit und vor allem dafür, daß er trotz seiner zahlreichen Verpflichtungen immer für mich Zeit fand, wenn ich mich an ihn wandte. Bei Herrn Prof. Dr. Rudolf Rengier bedanke ich mich für die zügige Erstellung des Zweitgutachtens.

Herrn Oberstaatsanwalt Glette aus Heidelberg und Herrn Lippok vom Kreisjugendamt des Rhein-Neckar-Kreises habe ich zu danken für die Hilfe, die sie mir im Rahmen der Aktenanalyse zuteil werden ließen. Ferner bedanke ich mich bei allen Richterinnen und Richtern, Staatsanwältinnen und Staatsanwälten sowie den Jugendgerichtshelferinnen und Jugendgerichtshelfern im Rhein-Neckar-Kreis für ihre Bereitschaft zur Mitwirkung an dieser Arbeit.

Meinem Ehemann danke ich für seine liebevolle Unterstützung.

In großer Dankbarkeit widme ich diese Arbeit meinen Eltern, Elisabeth und Helmut Engelberg. 


\section{Inhaltsverzeichnis I}

Kapitel 1: Einleitung............................................................................................. 1

Kapitel 2: Überblick über die historische Entwicklung des Jugendstrafrechts unter besonderer Berücksichtigung von Arbeitsweisungen und Arbeitsauflagen. 3

$\$ 1$ Die Entwicklung des Jugendstrafrechts bis zum Jugendgerichtsgesetz vom 16. Februar 1923 ..................................... 3

$\S 2$ Das Reichsjugendgerichtsgesetz vom 10. November 1943.................. 9

$\S 3 \quad$ Das JGG vom 04. August 1953 ................................................................... 11

\$ 4 Das Erste Gesetz zur Änderung des Jugendgerichtsgesetzes vom 30. August 1990 ..................................................................................... 13

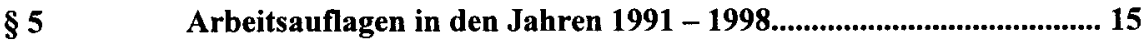

Kapitel 3: Die Unterscheidung zwischen Arbeitsweisungen und Arbeitsauflagen in der Theorie

\$ Das Wesen und die Voraussetzungen für die Anordnung der Arbeitsweisungen

\$2 Das Wesen und die Voraussetzungen für die Auferlegung der Arbeitsauflagen...................................................................................................... 26

\$ Die Verfassungsmäßigkeit von Arbeitsweisungen und Arbeitsauflagen im Hinblick auf Art. 12 GG 30

§ Ü̈berblick über die Differenzierungsansätze zwischen Arbeitsweisungen und Arbeitsauflagen in der Literatur

Kapitel 4: Der Stand der Forschung und die Beschreibung der eigenen empirischen Untersuchung................................................................60 60

$\S 1 \quad$ Ziel und Gegenstand der Untersuchung.............................................. 60

$\S 2$ Bisheriger Stand der Forschung...........................................................6 60 
$\S 3 \quad$ Die Untersuchungshypothesen..................................................................... 64

$\S 4 \quad$ Methodische Vorgehensweise......................................................................6 67

Kapitel 5: Darstellung der Ergebnisse der Untersuchung............................ 77

$\S 1 \quad$ Ergebnisse der Aktenanalyse................................................................. 77

§ 2 Ergebnisse der Interviews mit den Jugendrichtern und Staatsanwälten ......................................................................................... 151

$\S 3 \quad$ Ergebnisse der Befragung der Jugendgerichtshilfe............................. 172

§ $4 \quad$ Ergebnisse der Befragung der Institutionen.......................................... 185

\$ 5 Zusammenfassung der Ergebnisse der eigenen empirischen Untersuchung............................................................................................... 202

Kapitel 6: Schlußbetrachtungen und Ausblick............................................ 206

§1 Abschließender Vergleich der Praxis mit den theoretischen Abgrenzungsversuchen............................................................................. 206

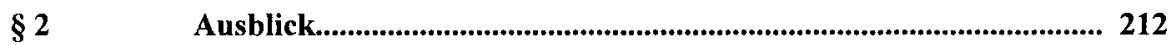




\section{Inhaltsverzeichnis II}

Abkürzungsverzeichnis ........................................................................................ XVII

Tabellenverzeichnis...................................................................................................... XXI

Schaubildverzeichnis ............................................................................................. XXIII

Kapitel 1: Einleitung.................................................................................... 1

Kapitel 2: Überblick über die historische Entwicklung des Jugendstrafrechts unter besonderer Berücksichtigung von Arbeitsweisungen und Arbeitsauflagen ............................... 3

§ 1 Die Entwicklung des Jugendstrafrechts bis zum Jugendgerichtsgesetz vom 16. Februar 1923 ........................................... 3

I. Constitutio Criminalis Carolina .......................................................... 3

II. Die Zeit bis zum Ende des 18. Jahrhunderts.......................................... 3

III. Das Preußische Allgemeine Landrecht (ALR) ........................................ 4

IV. Das deutsche Partikularrecht des 19. Jahrhunderts............................... 4

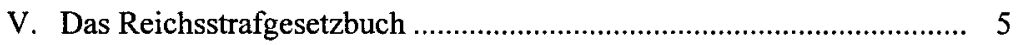

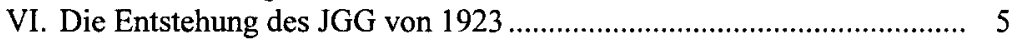

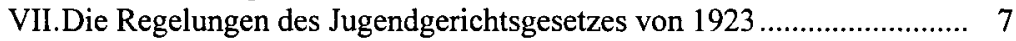

§ 2 Das Reichsjugendgerichtsgesetz vom 10. November 1943....................... 9

$\S 3$ Das JGG vom 04. August 1953 …................................................................ 11

§ 4 Das Erste Gesetz zur Änderung des Jugendgerichtsgesetzes vom 30. August 1990 .......................................................................................... 13

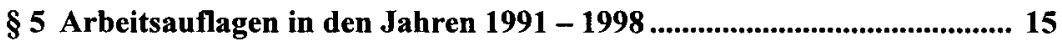

Kapitel 3: Die Unterscheidung zwischen Arbeitsweisungen und Arbeitsauflagen in der Theorie......................................................... 17

\$ 1 Das Wesen und die Voraussetzungen für die Anordnung der Arbeitsweisungen........................................................................................ 17

I. Zweck der Weisungen ........................................................................ 17

1. Allgemeines ..................................................................................... 17

2. Die Arbeitsweisungen gemäß § 10 Abs.1 S.3 Nr.4 JGG ................... 17

II. Anwendungsvoraussetzungen..................................................... 18

1. Schuldfähigkeit ............................................................................. 18

2. Die Straftat als Anlaß................................................................... 18 
3. Der Erziehungsgedanke im Rahmen der Weisungen ....................... 20

a) Erziehungsbedürftigkeit................................................................... 21

b) Erziehungsfähigkeit ..................................................................... 21

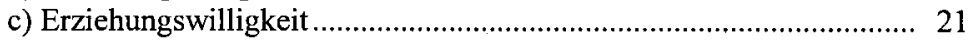

4. Bestimmtheit der Arbeitsweisungen ................................................ 22

5. Zumutbarkeit ................................................................................. 22

6. Sonstige Voraussetzungen .......................................................... 24

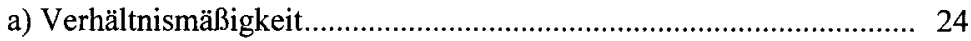

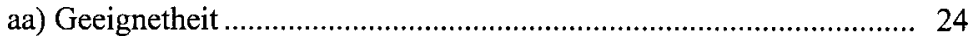

bb) Erforderlichkeit....................................................................... 24

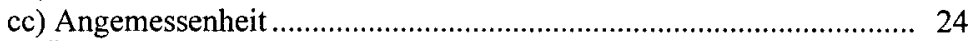

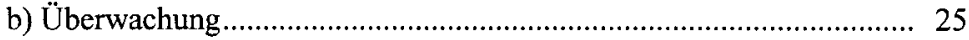

c) Weitere Anforderungen .......................................................... 25

\section{§ 2 Das Wesen und die Voraussetzungen für die Auferlegung} der Arbeitsauflagen ......................................................................................... 26

I. Zweck der Auflagen............................................................................... 26

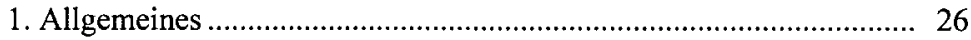

2. Die Arbeitsauflagen gemäß § 15 Abs.1 S.1 Nr.3............................ 27

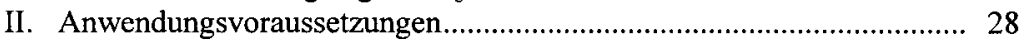

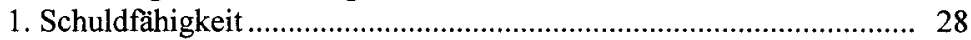

2. Nichtausreichen von Erziehungsmaßregeln................................... 28

3. Nichtgebotensein von Jugendstrafe ............................................... 28

a) Schädliche Neigung ...................................................................... 29

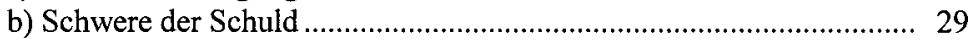

4. Weitere Voraussetzungen .......................................................... 30

a) Zumutbarkeit ........................................................................... 30

b) Verhältnismäßigkeit .............................................................. 30

\section{§ 3 Die Verfassungsmäßigkeit von Arbeitsweisungen und} Arbeitsauflagen im Hinblick auf Art. 12 GG............................................. 30

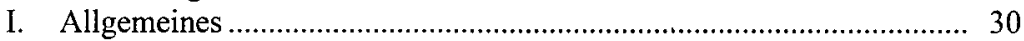

1. Schutzbereiche von Art. 12 Abs.2 und Abs.3 GG ........................ 30

2. Schranken von Arbeitszwang und Zwangsarbeit............................. 31

II. Verfassungsrechtliche Bedenken ....................................................... 32

1. Arbeitsweisungen und Art. 12 GG .............................................. 32

a) Zusammenfassung der Bundesverfassungsgerichtsentscheidung....... 32

b) Stellungnahme zu der Entscheidung .............................................. 33

2. Arbeitsauflagen und Art. 12 GG ............................................... 34

\section{\$ 4 Überblick über die Differenzierungsansätze zwischen}

Arbeitsweisungen und Arbeitsauflagen in der Literatur......................... 36

I. Erforderlichkeit einer theoretischen Abgrenzungsmöglichkeit ............. 36

II. Differenzierungsansätze....................................................................... 37 
1. Den Arbeitsweisungen kommt als alleiniger Zweck Erziehungsfunktion zu .................................................................. 37

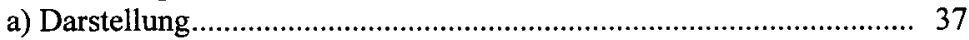

b) Diskussion ................................................................................... 39

2. Bei Arbeitsweisungen muß der Erziehungszweck im Vordergrund

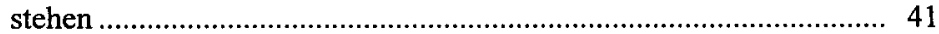

a) Darstellung........................................................................................ 41

b) Diskussion ........................................................................................ 42

3. Arbeitsweisungen regeln das Verhalten des Jugendlichen für einen längeren Zeitraum, Arbeitsauflagen erschöpfen sich in einer einmaligen Erfüllung ............................................................. 43

a) Darstellung...................................................................................... 43

b) Diskussion .................................................................................... 44

4. Arbeitsweisungen sind bei leichten Taten angebracht, Arbeitsauflagen hingegen bei mittelschwerer Kriminalität ................ 45

a) Darstellung................................................................................... 45

b) Diskussion ................................................................................. 46

5. Arbeitsweisungen sollen den Erziehungsprozeß selbst fördern ......... 48

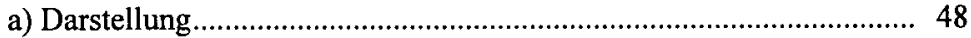

b) Diskussion ................................................................................... 48

6. Arbeitsauflagen richten sich, im Gegensatz zu Arbeitsweisungen, eher in die Vergangenheit ............................................................. 49

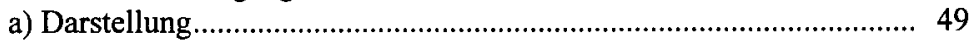

b) Diskussion ................................................................................. 50

7. Arbeitsweisungen sind Reaktion auf Defizite im Arbeitsbereich, die kriminalitätsfördernd sind ......................................................... 51

a) Darstellung........................................................................................ 51

b) Diskussion ............................................................................... 51

8. Arbeitsauflagen sollen im Gegensatz zu Arbeitsweisungen

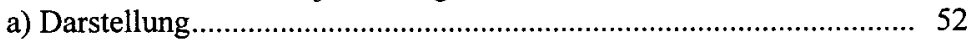

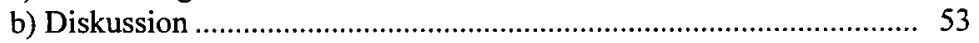

9. Arbeitsauflagen begründen eine sittliche Verpflichtung .................... 53

a) Darstellung................................................................................. 53

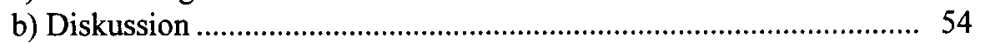

10. Abgrenzungsansatz von Itzel nach rein formalen Kriterien ............. 55

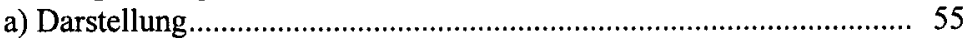

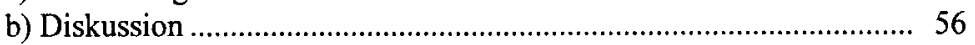

III. Zusammenfassung und Stellungnahme ........................................... 57 
Kapitel 4: Der Stand der Forschung und die Beschreibung der eigenen empirischen Untersuchung.

$\S 1$ Ziel und Gegenstand der Untersuchung .................................................6 60

§ 2 Bisheriger Stand der Forschung ................................................................. 60

§ 3 Die Untersuchungshypothesen ................................................................... 64

I. Unterscheidung anhand der Bezeichnung.............................................. 64

II. Unterscheidung anhand Tat und Täter............................................... 65

III. Unterscheidung nach der Einstellung zur Arbeit .................................... 66

IV. Abgrenzung anhand der Folgen der Tat ................................................. 66

V. Unterscheidung bei der Ableistung der Arbeitsleistung ......................... 66

\$ 4 Methodische Vorgehensweise....................................................................... 67

I. Darstellung der Untersuchungsformen und der

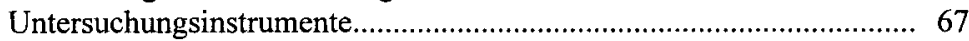

1. Die Aktenanalyse der Strafverfahrensakten ...................................... 67

2. Die Befragung der Richter und Staatsanwälte .................................. 68

3. Die Befragung der Mitarbeiter der Jugendgerichtshilfe ..................... 69

4. Die Befragung der Institutionen ........................................................ 69

II. Die Auswahl der untersuchten Fälle ................................................. 70

1. Die Aktenanalyse ........................................................................... 70

a) Räumliche und zeitliche Beschränkung............................................... 70

b) Beschränkung hinsichtlich der Anzahl .......................................... 71

c) Sonstige Beschränkungen ........................................................... 72

2. Die Interviews und die schriftliche Befragung ................................ 72

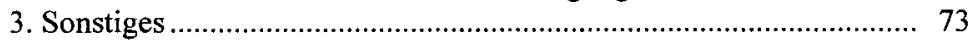

III. Durchführung der Untersuchung ……….......................................... 74

1. Die Aktenanalyse .......................................................................... 74

a) Vorbereitung des Vorhabens ......................................................... 74

b) Auswertung der Daten ............................................................... 74

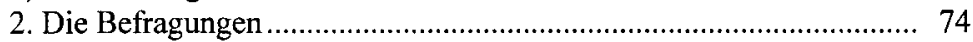

a) Die Richterinterviews .................................................................... 74

b) Die Interviews mit den zuständigen Jugendstaatsanwälten................ 75

c) Die Interviews mit den Jugendgerichtshelfern .................................. 75

d) Die Befragungen der Institutionen .................................................. 75

Kapitel 5: Darstellung der Ergebnisse der Untersuchung ......................... 77

§ 1 Ergebnisse der Aktenanalyse .................................................................. 77

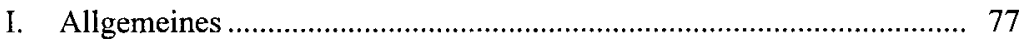

1. Die Einteilung in die Kategorien „Arbeitsweisungen“, "Arbeitsauflagen" und ,sonstige Arbeitsleistungen" 
2. Zeitlicher Rahmen.................................................................... 79

3. Eingeschaltete Behörden und Registerauszüge …….......................... 82

4. Strafantrag …................................................................................ 83

II. Kriterien zur Person des Täters und seinem Sozialbereich und ihr Einfluß auf die Art der Arbeitsmaßnahme ..................................... 83

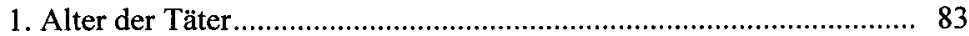

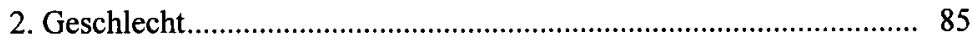

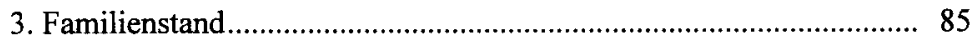

4. Nationalität und Geburtsort .......................................................... 86

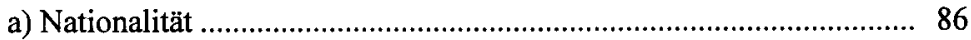

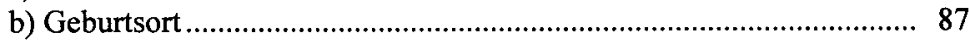

c) Erforderlichkeit des Einsatzes eines Dolmetschers .......................... 87

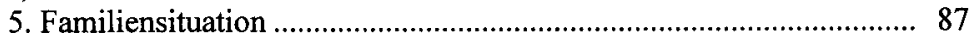

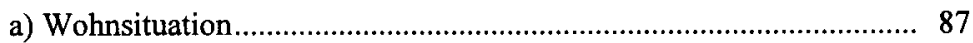

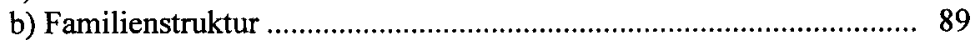

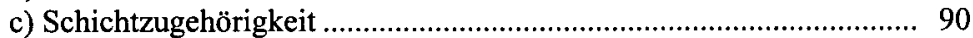

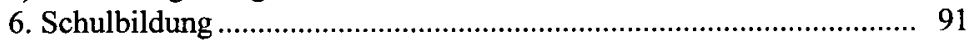

7. Tätigkeit im Zeitpunkt der Tat............................................................ 93

8. Einstellung zu Schule und Arbeit ....................................................... 94

9. Die finanzielle Situation des Täters .................................................. 95

a) Einkommen und Taschengeld........................................................ 96

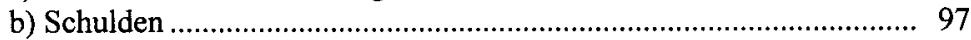

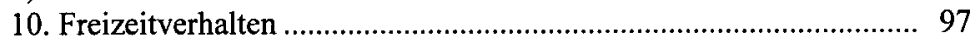

11. Auffälligkeiten mit Alkohol und Drogen......................................... 99

12. Anzahl der registrierten Vorstrafen und deren Sanktionierung ........ 99

a) Anzahl der Vorstrafen.................................................................... 99

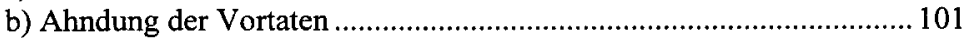

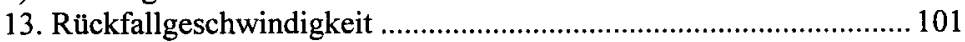

III. Einfluß der Tat auf die Art der Arbeitsmaßnahme ................................. 102

1. Einfluß der verletzten Normen auf die Sanktionsart .......................... 102

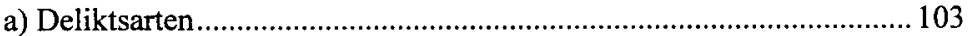

b) Übereinstimmung der Vortat mit der aktuellen Tat ............................ 107

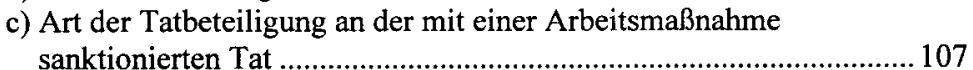

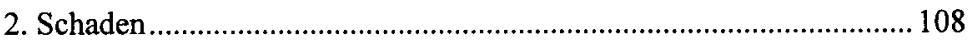

a) Art des Schadens......................................................................... 108

b) Verhältnis des Täters zu Opfer oder Geschädigten ............................. 109

c) Höhe des entstandenen Schadens bzw. Wert der durch die Tat erlangten Sache ................................................................................... 110

d) Art des Personenschadens ........................................................... 111

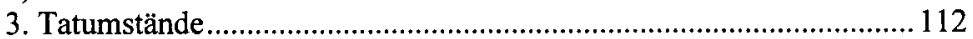

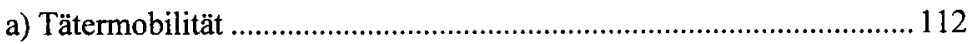

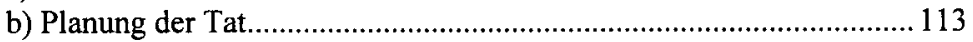

c) Alkohol- und Drogeneinfluß bei der Tatausübung ............................... 114 
III. Die polizeiliche Ermittlung und ihr Einfluß auf die Art der Arbeitsmaßnahme

1. Zur Informationsquelle über Tat und Tatverdacht..........................115

2. Die polizeiliche Vernehmung ................................................. 115

a) Stattfinden einer polizeilichen Vernehmung .................................. 116

b) Verhalten des Täters bei der Polizei .............................................. 116

c) Die Einstellung des Täters zur Tat bei der Polizei ............................. 117

d) Anwesenheit weiterer Personen bei der polizeilichen

3. Zeugen- und Geschädigtenvernehmung ......................................... 119

4. Weitere polizeiliche Maßnahmen .................................................. 119

V. Einstellung durch die Staatsanwaltschaft ....................................... 120

1. Anteile der Arbeitsmaßnahmen im informellen und formellen Verfahren ................................................................................. 120

2. Zur Stundenanzahl ................................................................. 121

3. Verbindung mit anderer strafrechtlicher Ahndung ......................... 122

4. Die Beteiligung der JGH bei Einstellungen durch die Staatsanwaltschaft................................................................... 122

a) Jugendgerichtshilfebericht im Diversionsverfahren ...................... 123

b) Vorschlag der Jugendgerichtshilfe bzgl. der Einstellung des Verfahrens............................................................................... 123

5. Berücksichtigung einer elterlichen Sanktion ................................. 124

VI. Entscheidung durch das Gericht .................................................. 125

1. Richterlich gewählte Abschlußform des Verfahrens ..................... 125

2. Einstellung gemäß § 47 JGG ...................................................... 126

3. Zuständigkeit des Gerichts......................................................... 126

4. Die Hauptverhandlung ............................................................. 126

a) Zeitliche Dauer bis zur Hauptverhandlung................................... 126

b) Besondere Verfahrensart des $\S 76$ JGG........................................ 127

c) Anwesenheit in der Hauptverhandlung von weiteren Personen neben dem Angeklagten............................................................. 128

d) Hauptverhandlung gegen mehrere Mitangeklagte......................... 130

e) Verhalten des Täters bei Gericht und seine subjektive Einstellung zur Tat ..................................................................................... 131

f) Zeugenvernehmung............................................................... 131

g) Anhörung der Eltern und elterliche Sanktion................................ 132

5. Rolle der Jugendgerichtshilfe in der mündlichen Verhandlung ......... 133

6. Anträge der Staatsanwaltschaft.................................................... 134

7. Anträge der Verteidiger ............................................................. 135

8. Tenor des Gerichts ..................................................................... 135

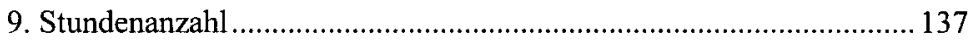

10. Zur weiteren jugendstrafrechtlichen Ahndung .............................. 138

VII. Arbeitsleistung und Durchführung .................................................... 139

1. Erste Anregung der Arbeitsleistung........................................ 139

2. Begründung zur gewählten Form der Arbeitsleistung .......................139

3. Fristsetzung für die Ableistung der Arbeitsleistung ...................... 140 
4. Probleme bei der Durchführung der Arbeitsleistung .......................... 141

5. Bezeichnung durch die Jugendgerichtshilfe und Institutionen ............ 142

6. Meldungen bzgl. der Ableistung der Arbeit ...................................... 142

VIII. Rechtsmittel, Registereintrag, Belehrung und Sonstiges..................... 143

1. Gescheiterte Diversionsangebote im Vorfeld bzgl. derselben Tat ....143

2. Belehrung gemäß Hauptverhandlungsprotokoll ................................. 143

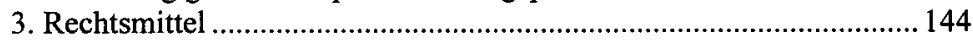

IX. Zusammenfassung der Ergebnisse der Aktenanalyse .......................... 144

\section{\$ 2 Ergebnisse der Interviews mit den Jugendrichtern und}

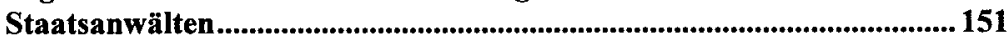

I. Interviews mit den Jugendrichtern......................................................... 151

1. Allgemeine Fragen ........................................................................... 151

2. Fragen zu Arbeitsweisungen und Arbeitsauflagen .............................. 152

3. Zusammenfassung der aus den Richterinterviews gewonnenen

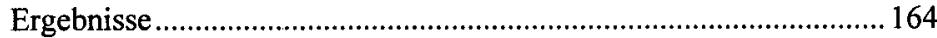

II. Interviews mit den Jugendstaatsanwälten.............................................. 167

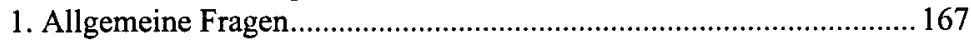

2. Fragen zu Arbeitsweisungen und Arbeitsauflagen .............................. 168

3. Zusammenfassung der aus den Interviews mit den Staatsanwälten gewonnenen Ergebnisse..................................................................... 171

\$ 3 Ergebnisse der Befragung der Jugendgerichtshilfe ................................... 172

I. Fragen zu Arbeitsweisungen und Arbeitsauflagen .................................. 172

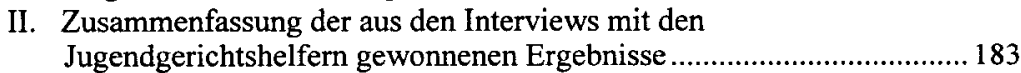

\$ 4 Ergebnisse der Befragung der Institutionen ................................................185

I. Fragen zur gemeinnützigen Arbeit ................................................... 185

1. Angebot an Einrichtungen .................................................................. 185

2. Angebot an möglichen Tätigkeiten .................................................. 187

3. Anzahl der Jugendlichen, die durchschnittlich pro Jahr in den

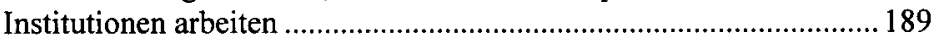

4. Informationen durch die Jugendgerichtshilfe ................................... 191

5. Kriterien zur Zuteilung der konkreten Arbeit .................................... 193

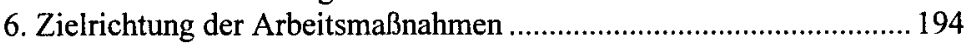

7. Probleme bei den Arbeitsmaßnahmen ............................................... 197

8. Beurteilung der Arbeitsmaßnahmen und Verbesserungsmöglichkeiten ........................................................... 198

II. Zusammenfassung der aus den Befragungen mit den Institutionen gewonnenen Ergebnisse

\section{§ 5 Zusammenfassung der Ergebnisse der eigenen empirischen}


XVI

Kapitel 6: Schlußbetrachtungen und Ausblick ............................................. 206

$\$ 1$ Abschließender Vergleich der Praxis mit den theoretischen Abgrenzungsversuchen ......................................................................................206

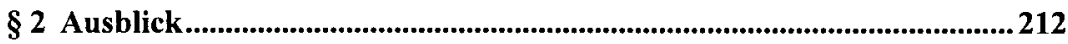

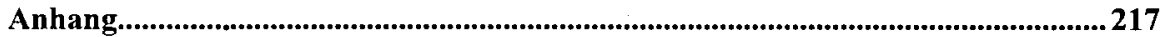

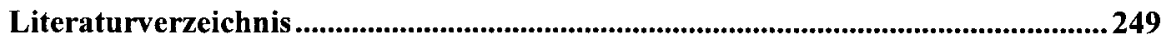




\section{Abkürzungsverzeichnis}

a.A. anderer Ansicht

ABM

Arbeitsbeschaffungsmaßnahmen

Abs.

Absatz

AKJGG Alternativkommentar zum Jugendgerichtsgesetz

ALR

Allgemeines Landrecht für die preußischen Staaten

Alt.

Alternative

Art.

Artikel

ASD

Allgemeiner Sozialer Dienst

AT

Allgemeiner Teil

Az.

Aktenzeichen

BayObLG Bayerisches Oberstes Landesgericht

Bem.

Bemerkung

BewHi

Bewährungshilfe

BGBl.

Bundesgesetzblatt

BGH

Bundesgerichtshof

BGHSt

BR

Entscheidungen des Bundesgerichtshofs in Strafsachen

BT

Bundesrat

BtMG

Bundestag

BVerfGE

Betäubungsmittelgesetz

BVerwGE

Entscheidungen des Bundesverfassungsgerichts

BVJ

bzgl.

Entscheidungen des Bundesverwaltungsgerichts

BZR

Berufsvorbereitungsjahr

bzw.

ca.

bezüglich

Bundeszentralregister

d.h.

ders.

beziehungsweise

df

circa

das heißt

derselbe

dies.

Freiheitsgrade

DM

dieselbe/n

Drs.

Deutsche Mark

DVJJ

Drucksache

e.V.

Deutsche Vereinigung für Jugendgerichte und Jugendgerichtshilfen

Einf. eingetragener Verein

EU Europäische Union

EuGRZ Europäische Grundrechte-Zeitschrift

$\mathrm{F}$

f/ff

Prüfgröße der Varianzanalyse

folgende

GA Goltdammer's Archiv für Strafrecht

GABl. Gemeinsames Amtsblatt des Landes Baden-Württemberg

GG Gundgesetz 
Grdl. z. Grundlagen zu

h.M. herrschende Meinung

Hrsg. Herausgeber

HS Halbsatz

i.d.R. in der Regel

i.S.d. im Sinne des

IKV Internationale Kriminalistische Vereinigung

JA Juristische Arbeitsblätter

JGG Jugendgerichtsgesetz

JGGÄndG Gesetz zur Änderung des Jugendgerichtsgesetzes

JGH Jugendgerichtshilfe

JR Juristische Rundschau

Js Registerzeichen der Ermittlungsverfahren in Strafsachen

JuS Juristische Schulung

JZ Juristenzeitung

KG Kammergericht

$\mathrm{km} \quad$ Kilometer

KrimJ Kriminologisches Journal

KZfSS Kölner Zeitschrift für Soziologie und Sozialpsychologie

lfd. laufende

Max Maximum

MDR Monatsschrift für Deutsches Recht

Min Minimum

MschrKrim Monatsschrift für Kriminologie und Strafrechtsreform

n absolute Zahl

NAM Neue ambulante Maßnahmen

NJW Neue Juristische Wochenschrift

Nr. Nummer

NStZ Neue Zeitschrift für Strafrecht

OLG Oberlandesgericht

OWi Ordnungswidrigkeit

OWiG Gesetz über Ordnungswidrigkeiten

$\mathrm{p}$

Wahrscheinlichkeit

PDV Polizeidienstvorschriften

PflVG Gesetz über die Pflichtversicherung für Kraftfahrzeughalter (Pflichtversicherungsgesetz)

RdJ Recht der Jugend (und des Bildungswesens)

RGBI. Reichsgesetzblatt

RJGG Reichsjugendgerichtsgesetz

RLJGG Richtlinien zum Jugendgerichtsgesetz

Rn. Randnummer

RStGB Reichsstrafgesetzbuch

S. Seite

StA Staatsanwaltschaft

Stddev Standardabweichung

StGB Strafgesetzbuch 


$\begin{array}{ll}\text { StPO } & \text { Strafprozeßordnung } \\ \text { StV } & \text { Strafverteidiger } \\ \text { StVG } & \text { Straßenverkehrsgesetz } \\ \text { u.a. } & \text { und andere / unter anderem } \\ \text { UJ } & \text { Unsere Jugend } \\ \text { vgl. } & \text { vergleiche } \\ \text { WaffG } & \text { Waffengesetz } \\ \text { z.B. } & \text { zum Beispiel } \\ \text { ZfJ } & \text { Zentralblatt für Jugendrecht (und Jugendwohlfahrt) / (Zentralblatt für } \\ & \text { Vormundschaftswesen, Jugendgerichte und Fürsorgeerziehung) } \\ \text { ZfS } & \text { Zeitschrift für Soziologie } \\ \text { ZRP } & \text { Zeitschrift für Rechtspolitik } \\ \text { ZStW } & \text { Zeitschrift für die gesamte Strafrechtswissenschaft }\end{array}$




\section{Tabellenverzeichnis}

Tabelle 1: Anzahl der nach Jugendstrafrecht Verurteilten, deren Straftaten mit Arbeitsauflagen in den Jahren 1991 - 1998 geahndet wurden ............... 16

Tabelle 2: $\quad$ Anzahl und Verteilung der Arbeitsmaßnahmen .................................... 78

Tabelle 3: Zeitraum von der Begehung der Tat bis zur ersten behördlichen

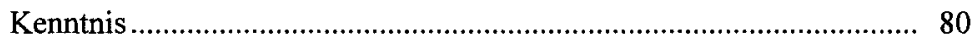

Tabelle 4: Zeitdauer von der Begehung der Tat bis zur Verfahrensbeendigung ..... 81

Tabelle 5: Zeitdauer von der ersten behördlichen Kenntnis bis zur Verfahrensbeendigung (..................................................................... 82

Tabelle 6: Alter im Zeitpunkt der Tatbegehung ................................................. 84

Tabelle 7: Nationalität der Täter ......................................................................... 86

Tabelle 8: Wohnort der Täter........................................................................... 88

Tabelle 9: Schulbildung der Täter............................................................ 92

Tabelle 10: Ausgeübte Tätigkeit der Täter …………............................................ 94

Tabelle 11: Einstellung der Täter zur Arbeit bzw. Schule .......................................... 95

Tabelle 12: Schulden der Täter ...................................................................... 97

Tabelle 13: Freizeitverhalten der Täter................................................................ 98

Tabelle 14: Anzahl der registrierten Vortaten ............................................................ 100

Tabelle 15: Rückfallgeschwindigkeit und zeitlicher Abstand zur jetzt verfolgten

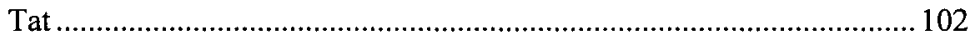

Tabelle 16: Verletzte Normen bei Diebstahlsdelikten ............................................ 104

Tabelle 17: Sonstige Vermögensdelikte nach dem StGB .......................................... 105

Tabelle 18: Sonstige Straftaten nach dem StGB ................................................... 105

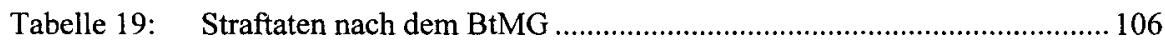

Tabelle 20: Sonstige, nicht nach dem StGB begangene Straftaten............................ 106

Tabelle 21: Höhe des durch die Tat verursachten Schadens.....................................111

Tabelle 22: Wert der durch die Tat erlangten Sache................................................. 111

Tabelle 23: Entfernung zwischen Wohnort und Tatort..............................................112

Tabelle 24: Verhalten des Täters bei der Polizei ..................................................... 117

Tabelle 25: Abschlußverfügung der Staatsanwaltschaft.......................................... 120

Tabelle 26: Häufigkeiten der von der Staatsanwaltschaft vorgeschlagenen und abgeleisteten Stundenanzahl ...................................................... 122

Tabelle 27: Abschlußform des gerichtlichen Verfahrens .......................................... 125

Tabelle 28: Zeitdauer von der ersten behördlichen Kenntnis bis zur Hauptverhandlung. 
Tabelle 29: Anzahl der vereinfachten Jugendverfahren gemäß $\S 76$ ff JGG............ 128

Tabelle 30: Anzahl der gerichtlich vernommenen Zeugen ..................................... 132

Tabelle 31: Häufigkeiten der richterlich verhängten Stundenanzahl......................... 137

Tabelle 32: Anzahl und Arten der Einrichtungen, in denen Arbeitsmaßnahmen

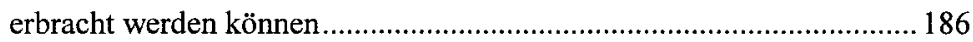

Tabelle 33: Zeitpunkt, seit dem die Einrichtungen Arbeitsmaßnahmen anbieten ..... 187

Tabelle 34: Arten der angebotenen Tätigkeiten.................................................... 188

Tabelle 35: Informationen für die Einrichtungen ................................................... 192

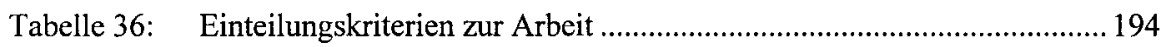

Tabelle 37: Zielrichtung der Arbeitsmaßnahmen .................................................. 195

Tabelle 38: Erzieherisches Element der Arbeitsmaßnahmen ................................... 195

Tabelle 39: Strafendes Element der Arbeitsmaßnahmen ....................................... 196

Tabelle 40: Beurteilung der Arbeitsmaßnahmen aus Sicht der Einrichtungen.......... 198 


\section{Schaubildverzeichnis}

Schaubild 1: Verteilung der Arbeitsmaßnahmen unter Zugrundelegung der Meldung an das BZR ................................................................. 78

Schaubild 2: Verteilung der Arbeitsmaßnahmen unter Zugrundelegung der von den Richtern und Staatsanwälten gewählten Bezeichnung............. 79

Schaubild 3: Verteilung des Alters der Täter im Zeitpunkt der Tatbegehung............ 84

Schaubild 4: Schulbildung der Täter..................................................................... 91

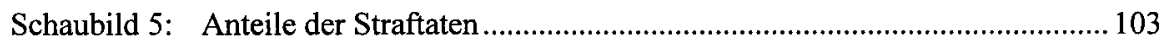

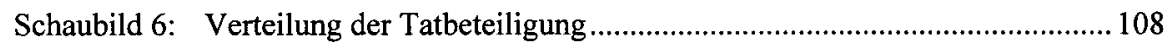

Schaubild 7: Vorliegen und Art des Schadens.......................................................... 109

Schaubild 8: Zeit zur Planung der Tat ........................................................................ 114

Schaubild 9: Bezeichnung der Arbeitsmaßnahmen durch die Staatsanwaltschaft im Rahmen von $\S 45 \mathrm{Abs.2} \mathrm{JGG} \mathrm{in} \mathrm{der} \mathrm{Abschlußverfügung} \mathrm{.................} 121$

Schaubild 10: Anwesenheit von weiteren Personen in der Hauptverhandlung .............. 130

Schaubild 11: Anträge der Staatsanwaltschaft........................................................... 135

Schaubild 12: Bezeichnung der Arbeitsmaßnahmen im Tenor .................................... 136

Schaubild 13: Verhältnis der Stundenzahlen bei zunehmendem Schaden bzw. Wert... 138

Schaubild 14: Durchschnittliche Anzahl jugendlicher Straftäter, die pro Jahr ihre Arbeitsmaßnahmen in den Einrichtungen absolvieren ............................ 190 